\title{
Miracles of molecular uniting
}

\author{
Qianqian $\mathrm{Li}^{1}$ and Zhen $\mathrm{Li}^{1,2^{*}}$
}

"Union is strength". In the realm of organic molecules, the macroscopic performance of molecular aggregates is not just the simple overlay of single molecules, and in many cases, new properties can be created by molecular uniting with particular packing modes [1-4]. As to the organic emissive materials, various changes can be realized from single molecules to aggregates (Fig. 1), including the emerged bright emission, the varied emission color, the different emission forms, and the arisen new excitation processes.

\section{AGGREGATION-INDUCED EMISSION (AIE)}

The luminogens with the AIE characteristic are nonluminescent or weakly luminescent in dilute solution but emit intensely in the aggregate state, opposite to the common aggregation-caused quenching (ACQ) effect $[5,6]$. Normally, AIEgens are organic $\pi$-systems with twisted configurations, which are beneficial to the molecular rotation and vibration as the nonradiative process in solution, leading to the nearly dark state in isolated conditions. The molecular motions and severe $\pi-\pi$ interactions as the nonradiative decay channels can be blocked in the aggregate state due to relatively loose molecular packing, spatial constraint and intermolecular interactions, while in solid state or nanoparticle, the radiative decay of excited state will dominate, enabling the efficient emission of AIEgens (Fig. 2). These intriguing luminescent phenomena are of great importance for practical applications in optoelectronic devices and bioimaging [7-9], because of their high solid-state photoluminescent quantum yields (PLQYs) and dynamic emissive property with "turn-on" response to aggregate states, together with proper solubility or molecular behavior in water or hydrophilic system.

Actually, the AIE phenomenon is triggered by the formation of molecular aggregates, but not the single molecules. More importantly, AIE affords a unique op-

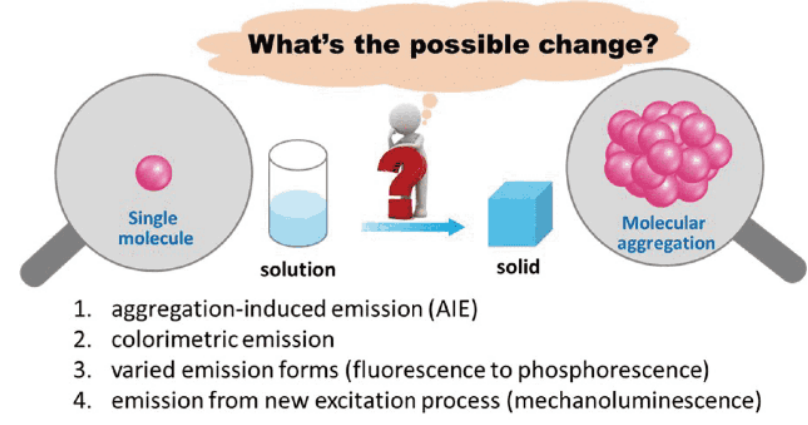

Figure 1 The proposed change of emissive behaviors from solution (single molecule) to solid (molecular aggregate).

portunity to deeply understand the internal mechanism of photoluminescence process, with more attention being attracted into the molecular packing in the aggregate state as the luminescent source. Accordingly, some novel emissive forms and materials have been explored or recalled new lives in recent years, for instance, the organic room temperature phosphorescence (RTP), mechanoluminescence $(\mathrm{ML})$, excimer emission, fluorescence of non-aromatic organic systems, and so on. All of them exhibit much distinguishing emissive properties in isolated and aggregate states, further demonstrating the power of molecular uniting.

Basically, most of light emission materials consist of various aromatic rings with different linkage modes, since they can form the rigid $\pi$-system to stabilize the excited states. While the flexible chains are normally considered as the nonluminous materials due to the actively molecular motions with low energy barriers and absence of conjugated systems. A few years ago, this common sense was broken by the bright fluorescence of some non-aromatic polymers and organic molecules, including poly(amido amine) (PAMAM), hyperbranched poly(amineester)s (HypETs), polyurea (PURE) dendrimers, branched PEIs, polyacrylonitrile, hyperbranched polysiloxanes

\footnotetext{
${ }^{1}$ Sauvage Center for Molecular Sciences, Department of Chemistry, Wuhan University, Wuhan 430072, China

${ }^{2}$ Institute of Molecular Aggregation Science, Tianjin University, Tianjin 300072, China

* Corresponding author (emails: lizhen@whu.edu.cn or lizhentju@tju.edu.cn)
} 

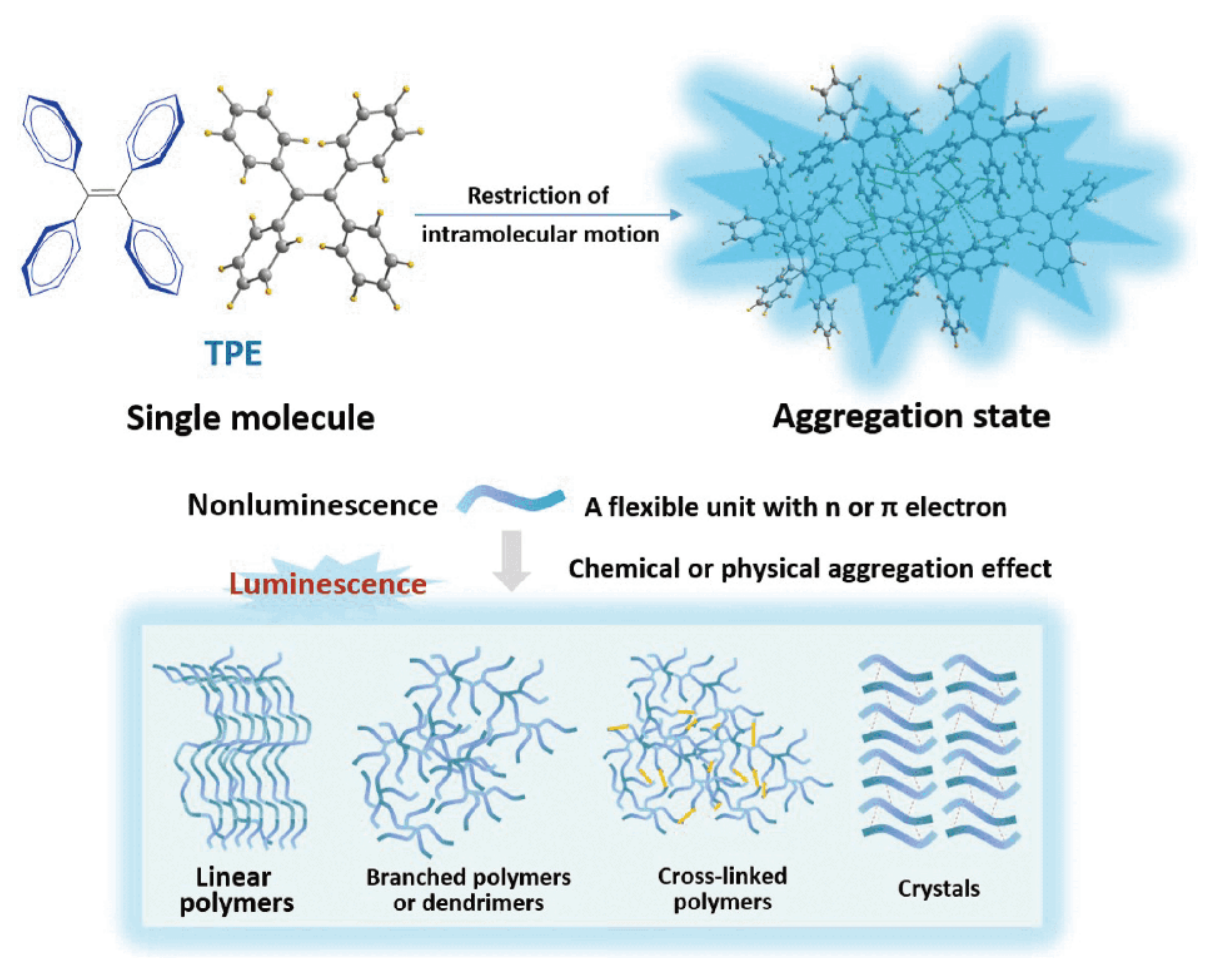

Figure 2 The AIE effect in organic molecules and CTE of non-aromatic luminous system.

(HBPSi), and natural biopolymers (e.g., cellulose, starch, peptide) (Fig. 2) [10,11]. The common feature of these systems is the essential components of atoms with lone pair electrons, for instance, $\mathrm{N}, \mathrm{O}, \mathrm{S}$, and $\mathrm{P}$ atoms, and/or $\pi$ electrons from carbonyl groups, carbon-carbon double bonds, etc. With the optimized linear or branched structures, the tight molecular packing aggregate of the key components was induced by the reasonably covalent linkage as fixed modes with tunable conformation, and thus, the inter- or intra-chain interactions from hydrogen bonding, $\pi-\pi$ and/or $n-\pi$ coupling can be formed and contribute much to the electron delocalization and rigid conformation of the non-aromatic system, resulting in the light emission in aggregate state. This kind of luminescence is sensitive to the topological structures, molecular weight and surrounding environments of the whole organic system because the dynamic interactions among key units can be further improved by the sequential crosslinking or self-assembling process [12,13]. Moreover, the emission with different colors can be achieved in the same system by varied excitation wavelengths, itemed as excitation-dependent fluorescence emission (EDF), mainly due to the various clusters of key components, and cluster-triggering emission (CTE) is considered as the main mechanism for these non-aromatic luminous system $[14,15]$.

\section{Colorimetric emission}

For the emission of nonaromatic systems, the key point is the formation of relatively stable excitation state as we emphasized previously [3]. Once the relatively stable excitation states are formed, the emission could be emitted through the radiative route in molecular aggregates, which can well explain the dramatically enhanced emission of pyrene from single molecules to aggregate, regardless of its big planar system without any rotor or motion moieties commonly present in AIEgens. In detail, compared with the weak emission of single pyrene molecule in the UV region, a red-shifted and broad emission band at $450-500 \mathrm{~nm}$ could be observed in pyrene crystal for the formation of excimer [16,17]. Mostly, excimerformation involves the excitation of ground-state dimer into its locally excited (LE) state, which then forms an excimer via molecular rearrangement, and excitation process can be facilitated by the pronounced $\pi-\pi$ interactions of adjacent molecules stacked in a cofacial arrangement (Fig. 3). Similar phenomena can be observed in merocyanine dyes with $\mathrm{H}$ aggregates and some cyano- 


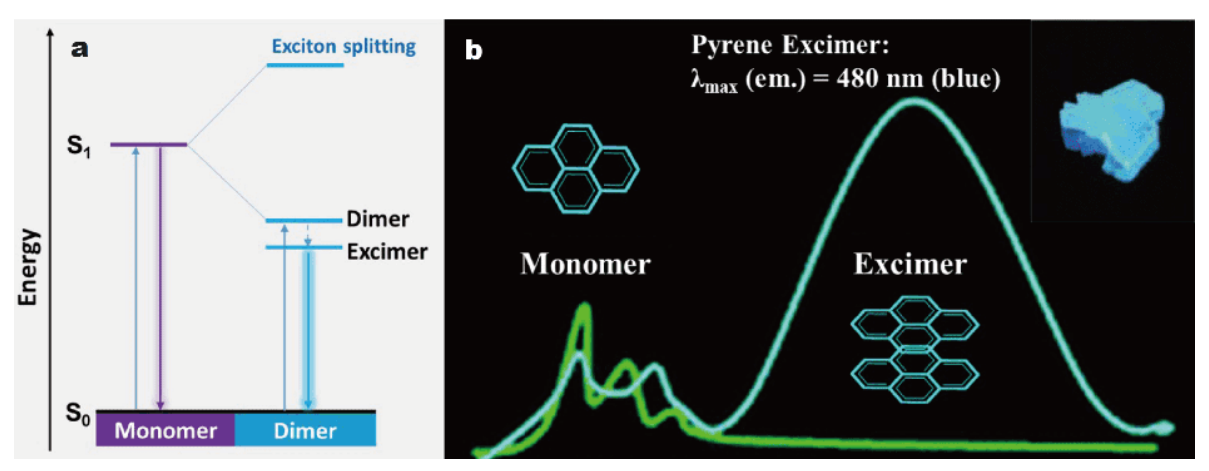

Figure 3 (a) Schematic diagram in terms of monomer and excimer emission, (b) packing mode of pyrene and the strong fluorescence for excimer formation. Reproduced with permission from Ref. [12]. Copyright 2009, American Chemical Society.

substituted compounds with compact molecular packing, as well as other aromatic hydrocarbons with van der Waals (vdW) dimers [18-20]. These colorimetric variations of emission with enhanced intensity can be applied into the sensing schemes, which are sensitive to the relative positions of these moieties under different conditions $[21,22]$.

\section{Varied emission forms}

Apart from the varied intensity and emission color from single molecules to aggregate state, sometimes, new luminescence forms can be generated in these molecular aggregating processes. In the last decade, the persistent RTP of pure organic molecules was an emerging phenomenon in the solid state as an important breakthrough (Fig. 4a), since it is challenging for organic materials to overcome the spin-prohibition between single and triplet excited state, and achieve the stable triplet excited state [23-25]. Among various strategies for molecular design and engineering, the adjustment of molecular packing and intermolecular interactions is an efficient strategy to improve the RTP effect of organic materials (Fig. $4 \mathrm{~b}$ ). Basically, the strong molecule-molecule interactions, such as halogen bonding and hydrogen bonding, and the crystal lattice are beneficial to the restriction of molecular motions, which can inhibit the possible non-radiative transition to stabilize the triplet excitons [26-28]. Through the systematical investigation of the relation between molecular packing and RTP effect, the face-toface packing mode is considered as the one which can stabilize triplet excitons by the generation of long-lived triplet excited states [29-31].

For instance, the RTP lifetimes of the 10-phenyl-10Hphenothiazine 5,5-dioxide based luminogens increase from $88\left(\mathrm{CS}-\mathrm{CH}_{3} \mathrm{O}\right)$ to $188 \mathrm{~ms}(\mathrm{CS}-\mathrm{H})$, then to $410 \mathrm{~ms}$ (CS-F), accompanying with the decreased centroid-cen- troid distances between two adjacent benzenes from 4.251 to $3.773 \AA$, as well as their reduced dihedral angles from $30.03^{\circ}$ to $0^{\circ}$ (Fig. $4 \mathrm{~b}$ ) [32]. The substituents with different electronic properties can adjust the molecular packing, and the electron-withdrawing substituents can effectively reduce the electron cloud density of $\pi$-system and decrease the repulsion between the adjacent molecules, which achieves closer molecular packing to enhance the $\pi-\pi$ interactions, facilitating the ultralong RTP effect. This can be further confirmed by the carbazole-based system (Fig. 4c) [29]. However, the intermolecular interactions and packing modes are hard to be controlled in crystals because of the complicated relationship between molecular packing and chemical structures. Recently, assembly was incorporated as an additional force to suppress the molecular motions and optimize molecular arrangement [33,34]. Accordingly, various approaches have been attempted, including co-crystallization, hostguest system, and dopant-based system, and so on, which expand the field of organic RTP, in favor of their applications spanning from optoelectronic to photomedicine [35-37].

Similar to the case of fluorescence, despite the fact that to date a large number of investigations have focused primarily on RTP mechanisms of organic conjugated system bearing aromatic moieties, some abnormal materials with obvious RTP have emerged, for instance, carbon dots, biomacromolecules and some non-aromatic polymers [38]. For example, cyanoacetic acid (CAA), an ultra-simple molecule without any aromatic moiety, was found to show RTP lifetime as long as $862 \mathrm{~ms}$ in the crystalline state by Fang et al. [39] (Fig. 4d). Through careful analyses of the single crystal, assembled layer by layer structure and multiple hydrogen bonding are proved as the main factors to increase the molecular rigidity and restrict the non-radiative transition, resulting 


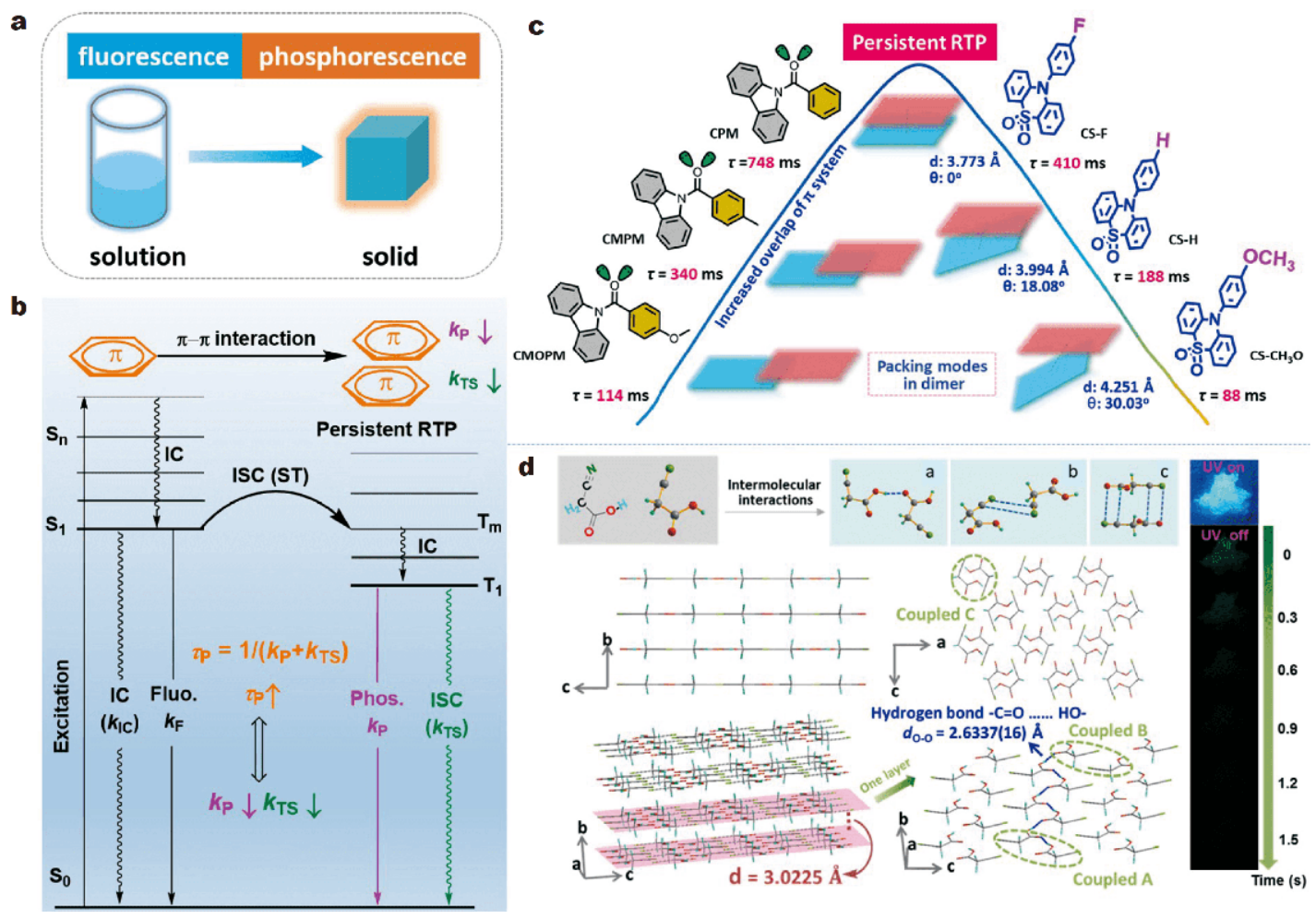

Figure 4 (a) The changed emission forms from single molecule to aggregate state. (b) The proposed mechanism for organic persistent RTP: the strong $\pi-\pi$ interaction could decrease the radiative transition $\left(k_{\mathrm{P}}\right)$ and non-radiative transition $\left(k_{\mathrm{TS}}\right)$ from $\mathrm{T}_{1}$ to $\mathrm{S}_{0}$ state, thus achieving the persistent RTP. Printed with permission from Ref. [32]. Copyright 2018, Nature Publishing Group. (c) The relationship between molecular packing modes and RTP properties. (d) RTP effect of organic systems without aromatic rings. Printed with permission from Ref. [39]. Copyright 2018, Royal Society of Chemistry.

in the radiative decay as persistent RTP. It further confirms the distinguishing emissive behavior originating from the optimized aggregate state, in addition to the chemical structure of organic molecules.

\section{Emission from new excitation process}

More interestingly, light emission from new excitation process can be realized in molecular aggregates. Unlike the normal luminescence from irradiation or electric driving, some organic solids exhibit attractive emissions upon mechanical stimuli, defined as ML or triboluminescence (TL) [40,41]. Generally, the multi-mechanosensitivity of certain ML materials is related to the lattice behavior, such as the molecular arrangement in the solid state. According to the intensive investigation on organic ML materials, the stuck packing mode is the key factor, in addition to the piezoelectric effect, since the possible molecular slippage under the mechanical force is the main non-radiative process (Fig. 5a) [42-44]. Similar to photoluminescence (PL), ML can be fluorescence or phosphorescence under different conditions, mainly re- lated to the electronic property of the excited state generated by mechanical stimuli. And the first example of fluorescence-phosphorescence dual ML in organic materials was realized by 2 -([1,1':3',1"-terphenyl] - $5^{\prime}$-yl)-4,4,5, 5-tetramethyl-1,3,2-dioxaborolane (DPP-BO), which only exhibited fluorescence under irradiation as $\mathrm{PL}$ emission (Fig. 5b) [45].

Thus, PL and ML have some similarity for the common radiative transition from excited state to ground state, but the excited state may be different for varied stimulations and molecular arrangements. Moreover, the ML effect is dynamic in some cases, mainly dependent on varied molecular configures or intermolecular interactions under the continuous mechanical stimulus. As to phenothiazine derivative, compound FCO-CzS shows changeable mechanoluminescent emissions from blue to white and yellow, accompanying with the molecular conformation transition of the phenothiazine moiety from quasi-axial to quasi-equatorial form (Fig. 5c) [46]. With the incorporation of multiple bromine atoms into fluorine unit, the resultant compound $\mathrm{BrFlu}-\mathrm{Br}$ also 

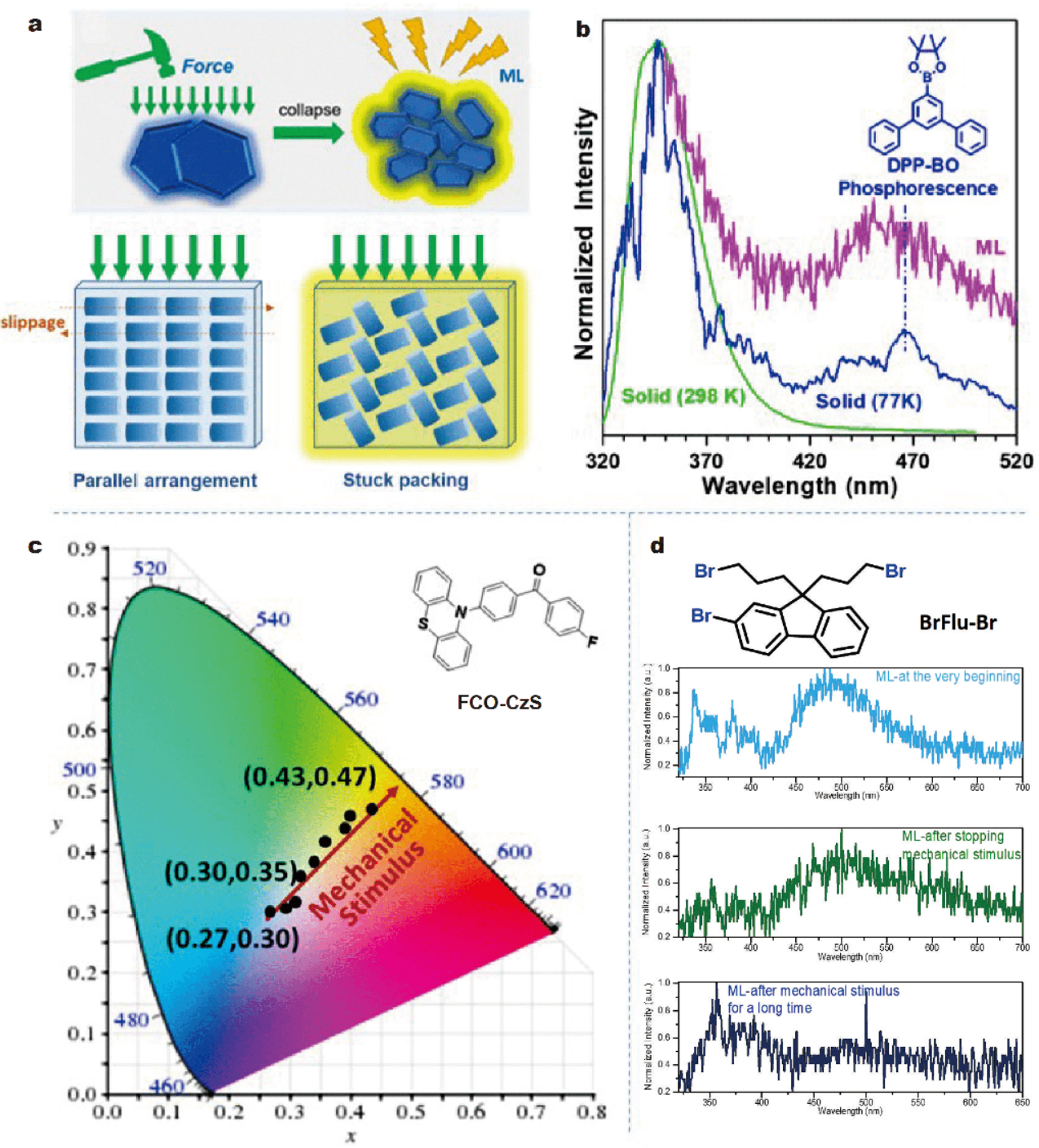

Figure 5 (a) The crucial role of molecular packing to ML property; (b) dual fluorescence-phosphorescence ML by DPP-BO. Printed with permission from Ref. [45]. Copyright 2016, Wiley. (c) The dynamic ML by FCO-CzS with the emission color changing from blue to white, then to yellow over grinding times. Printed with permission from Ref. [46]. Copyright 2018, Wiley. (d) The dynamic ML by BrFlu-Br for the varied intermolecular interactions. Printed with permission from Ref. [47]. Copyright 2018, Wiley.

shows tri-color ML switching, which is closely related to the variational intermolecular $\mathrm{Br} \cdots \mathrm{Br}$ interactions during mechanical stimulus (Fig. 5d) [47].

In summary, the emissive behaviors of organic molecules experience the interesting transformation from single molecule to aggregate state, which can be considered as the uniting effect by molecular packing. The intermolecular interactions should be considered as one of the main elements to the molecular arrangement and orientation in the aggregate state, which can be affected by many factors, such as the size, structure and electronic nature of the organic molecules. Basically, for the aro- matic systems, the small, unsubstituted aromatic moieties preferred edge-to-face packing modes, whereas substituted and large multiring aromatic compounds prefer parallel displaced geometry [48]. Apart from conjugated skeletons, the minor changes in side chains, for instance, the length or branching structure of alkyl groups, can make huge alterations in packing modes, as partially demonstrated in the corresponding single crystals, directly resulting in the different luminescence properties generated in the solid state. For instance, when the N-substituents in phenothiazine 5,5-dioxide derivatives are replaced by various alkyl chains, the odd-even effect of 
alkanes can be realized in the RTP properties of the resultant CS- $\mathrm{C}_{n} \mathrm{H}_{2 n+1}$ series, mainly due to the different $\pi-\pi$ interactions with parallel arrangement by various side chains [49].

These interactions are more complex in the non-aromatic system, since the whole structures are more flexible and changeable, and thus they are hard to be controlled accurately by molecular design with the cooperation of key units with lone pair and/or $\pi$ electrons, as well as the topological structure of dendrimers and polymers. Although non-aromatic systems demonstrate better biocompatibility and less toxicity in comparison with aromatic ones, some issues still exist in colorimetric purity and luminescent efficiency for the dynamic and varied molecular aggregates. Therefore, more efforts are consequently essential to control the aggregation behavior of these complicated systems to optimize their emission properties.

Thus, with the rational design of organic molecules to form special molecular packing in the aggregate state, various emissions with different forms and excitation processes can be generated by the joint efforts of molecules with multiple interactions. These non-covalent interactions among organic molecules exhibit flexible forms without fixed directions and strengths, which are more complex than that of the covalent bonding and afford more possibilities, resulting in the generation of new emission behaviors by the power of molecular uniting. So far, although the behavior of molecular aggregates could not be controlled and well understood completely, the much or totally different properties have been recognized from single molecules to molecular aggregates, clearly showing the power of molecular aggregate. Accordingly, our recognition should not only be limited on the level of single molecule, but turned to molecular aggregates with the Molecular Uniting Set Identified Characteristic (MUSIC).

"MUSIC" in the microscopic molecule world is similar to the music in our lives: basic atoms are just like different notes, and the melody with the alignment of different notes are similar to the organic moieties constructed by covalent linkage of different atoms with specific sequences. Correspondingly, the MUSICs, which are heavily dependent on the molecular uniting with different packing modes, resemble the symphony with the coming together of music voiced by different instruments. Through the optimized modulation of molecular structures and packing modes, the characteristic of molecular uniting can achieve excellent performance as a grand symphony. And actually, MUSIC is not only lim- ited to luminescence materials, but widely applicable to other functional materials such as organic field-effect transistors and organic photovoltaic devices [50,51]. In one word, the time of MUSIC is coming, with miracles of molecular uniting.

Received 24 July 2019; accepted 27 August 2019;

published online 16 September 2019

1 Mei J, Leung NLC, Kwok RTK, et al. Aggregation-induced emission: Together we shine, united we soar! Chem Rev, 2015, 115: 11718-11940

2 Jiang N, Shen T, Sun JZ, et al. Aggregation-induced emission: right there shining. Sci China Mater, 2019, 62: 1227-1235

3 Li Q, Li Z. The strong light-emission materials in the aggregated state: What happens from a single molecule to the collective group. Adv Sci, 2017, 4: 1600484

4 Li J, Shen P, Zhao Z, et al. Through-space conjugation: A thriving alternative for optoelectronic materials. CCS Chem, 2019, 1: 181196

5 Feng G, Liu B. Aggregation-induced emission (AIE) dots: Emerging theranostic nanolights. Acc Chem Res, 2018, 51: 1404-1414

6 Mei J, Hong Y, Lam JWY, et al. Aggregation-induced emission: The whole is more brilliant than the parts. Adv Mater, 2014, 26: 5429-5479

7 Yang J, Chi Z, Zhu W, et al. Aggregation-induced emission: a coming-of-age ceremony at the age of eighteen. Sci China Chem, 2019, 62: 1090-1098

8 Fu W, Yan C, Guo Z, et al. Rational design of near-infrared aggregation-induced-emission-active probes: In situ mapping of amyloid- $\beta$ plaques with ultrasensitivity and high-fidelity. J Am Chem Soc, 2019, 141: 3171-3177

9 Shao A, Xie Y, Zhu S, et al. Far-red and near-IR AIE-active fluorescent organic nanoprobes with enhanced tumor-targeting efficacy: Shape-specific effects. Angew Chem Int Ed, 2015, 54: 7275-7280

10 Chen $\mathrm{X}$, Luo W, Ma $\mathrm{H}$, et al. Prevalent intrinsic emission from nonaromatic amino acids and poly(amino acids). Sci China Chem, 2018, 61: 351-359

11 Zhou Q, Cao B, Zhu C, et al. Clustering-triggered emission of nonconjugated polyacrylonitrile. Small, 2016, 12: 6586-6592

12 Zhu S, Song Y, Shao J, et al. Non-conjugated polymer dots with crosslink-enhanced emission in the absence of fluorophore units. Angew Chem Int Ed, 2015, 54: 14626-14637

13 Geng T, Feng T, Ma Z, et al. Insights into supramolecular-interaction-regulated piezochromic carbonized polymer dots. Nanoscale, 2019, 11: 5072-5079

14 Zhou X, Luo W, Nie H, et al. Oligo(maleic anhydride)s: a platform for unveiling the mechanism of clusteroluminescence of nonaromatic polymers. J Mater Chem C, 2017, 5: 4775-4779

15 Zhao E, Lam JWY, Meng L, et al. Poly[(maleic anhydride)-alt(vinyl acetate)]: A pure oxygenic nonconjugated macromolecule with strong light emission and solvatochromic effect. Macromolecules, 2015, 48: 64-71

16 Figueira-Duarte TM, Müllen K. Pyrene-based materials for organic electronics. Chem Rev, 2011, 111: 7260-7314

17 Jung HS, Park M, Han DY, et al. $\mathrm{Cu}^{2+}$ ion-induced self-assembly of pyrenylquinoline with a pyrenyl excimer formation. Org Lett, 2009, 11: 3378-3381 
18 Han G, Kim D, Park Y, et al. Excimers beyond pyrene: A far-red optical proximity reporter and its application to the label-free detection of DNA. Angew Chem Int Ed, 2015, 54: 3912-3916

19 Saigusa H, Lim EC. Excimer formation in van der Waals dimers and clusters of aromatic molecules. Acc Chem Res, 1996, 29: 171178

20 Winnik FM. Photophysics of preassociated pyrenes in aqueous polymer solutions and in other organized media. Chem Rev, 1993, 93: $587-614$

21 Østergaard ME, Hrdlicka PJ. Pyrene-functionalized oligonucleotides and locked nucleic acids (LNAs): Tools for fundamental research, diagnostics, and nanotechnology. Chem Soc Rev, 2011, 40: 5771-5788

22 Kim JS, Quang DT. Calixarene-derived fluorescent probes. Chem Rev, 2007, 107: 3780-3799

23 Kenry, Chen C, Liu B. Enhancing the performance of pure organic room-temperature phosphorescent luminophores. Nat Commun, 2019, 10: 2111

24 Hirata S. Roles of localized electronic structures caused by $\pi$ degeneracy due to highly symmetric heavy atom-free conjugated molecular crystals leading to efficient persistent room-temperature phosphorescence. Adv Sci, 2019, 395: 1900410

25 Li Q, Tang Y, Hu W, et al. Fluorescence of nonaromatic organic systems and room temperature phosphorescence of organic luminogens: The intrinsic principle and recent progress. Small, 2018, 14: 1801560

26 Chai Z, Wang C, Wang J, et al. Abnormal room temperature phosphorescence of purely organic boron-containing compounds: the relationship between the emissive behaviorand the molecular packing, and the potential related applications. Chem Sci, 2017, 8: 8336-8344

27 Xiao L, Fu H. Enhanced room-temperature phosphorescence through intermolecular halogen/hydrogen bonding. Chem Eur J, 2019, 25: 714-723

28 Ogoshi T, Tsuchida H, Kakuta T, et al. Ultralong room-temperature phosphorescence from amorphous polymer poly(styrene sulfonic acid) in air in the dry solid state. Adv Funct Mater, 2018, 28: 1707369

29 Xie Y, Ge Y, Peng Q, et al. How the molecular packing affects the room temperature phosphorescence in pure organic compounds: Ingenious molecular design, detailed crystal analysis, and rational theoretical calculations. Adv Mater, 2017, 29: 1606829

30 An Z, Zheng C, Tao Y, et al. Stabilizing triplet excited states for ultralong organic phosphorescence. Nat Mater, 2015, 14: 685-690

31 Yang J, Ren Z, Chen B, et al. Three polymorphs of one luminogen: how the molecular packing affects the RTP and AIE properties? J Mater Chem C, 2017, 5: 9242-9246

32 Yang J, Zhen X, Wang B, et al. The influence of the molecular packing on the room temperature phosphorescence of purely organic luminogens. Nat Commun, 2018, 9: 840

33 Ma X, Wang J, Tian H. Assembling-induced emission: An efficient approach for amorphous metal-free organic emitting materials with room-temperature phosphorescence. Acc Chem Res, 2019, 52: 738-748

34 Kishimura A, Yamashita T, Yamaguchi K, et al. Rewritable phosphorescent paper by the control of competing kinetic and thermodynamic self-assembling events. Nat Mater, 2005, 4: 546-549

35 Bolton O, Lee K, Kim HJ, et al. Activating efficient phosphorescence from purely organic materials by crystal design. Nat Chem, 2011, 3: 205-210
36 Kabe R, Adachi C. Organic long persistent luminescence. Nature, 2017, 550: 384-387

37 Lee D, Bolton O, Kim BC, et al. Room temperature phosphorescence of metal-free organic materials in amorphous polymer matrices. J Am Chem Soc, 2013, 135: 6325-6329

38 Wang D, Wang $\mathrm{X}, \mathrm{Xu} \mathrm{C}$, et al. A novel metal-free amorphous room-temperature phosphorescent polymer without conjugation. Sci China Chem, 2019, 62: 430-433

39 Fang M, Yang J, Xiang X, et al. Unexpected room-temperature phosphorescence from a non-aromatic, low molecular weight, pure organic molecule through the intermolecular hydrogen bond. Mater Chem Front, 2018, 2: 2124-2129

40 Xie Y, Li Z. Triboluminescence: Recalling interest and new aspects. Chem, 2018, 4: 943-971

41 Mukherjee S, Thilagar P. Renaissance of organic triboluminescent materials. Angew Chem Int Ed, 2019, 58: 7922-7932

$42 \mathrm{Xu} \mathrm{B}, \mathrm{Li} \mathrm{W}, \mathrm{He} \mathrm{J}$, et al. Achieving very bright mechanoluminescence from purely organic luminophores with aggregation-induced emission by crystal design. Chem Sci, 2016, 7: 53075312

43 Wang $\mathrm{C}, \mathrm{Xu} \mathrm{B}, \mathrm{Li} \mathrm{M}$, et al. A stable tetraphenylethene derivative: aggregation-induced emission, different crystalline polymorphs, and totally different mechanoluminescence properties. Mater Horiz, 2016, 3: 220-225

44 Yu Y, Wang C, Wei Y, et al. Halogen-containing TPA-based luminogens: Different molecular packing and different mechanoluminescence. Adv Opt Mater, 2019, 44: 1900505

45 Yang J, Ren Z, Xie Z, et al. AIEgen with fluorescence-phosphorescence dual mechanoluminescence at room temperature. Angew Chem Int Ed, 2017, 56: 880-884

46 Yang J, Qin J, Geng P, et al. Molecular conformation-dependent mechanoluminescence: Same mechanical stimulus but different emissive color over time. Angew Chem Int Ed, 2018, 57: 1417414178

47 Wang J, Wang C, Gong Y, et al. Bromine-substituted fluorene: Molecular structure, $\mathrm{Br}-\mathrm{Br}$ interactions, room-temperature phosphorescence, and tricolor triboluminescence. Angew Chem Int Ed, 2018, 57: 16821-16826

48 Yao ZF, Wang JY, Pei J. Control of $\pi-\pi$ stacking via crystal engineering in organic conjugated small molecule crystals. Cryst Growth Des, 2018, 18: 7-15

49 Yang J, Gao H, Wang Y, et al. The odd-even effect of alkyl chain in organic room temperature phosphorescence luminogens and the corresponding in vivo imaging. Mater Chem Front, 2019, 3: 13911397

50 Sun L, Wang Y, Yang F, et al. Cocrystal engineering: A collaborative strategy toward functional materials. Adv Mater, 2019, 112: 1902328

51 Huang Y, Wang Z, Chen Z, et al. Organic cocrystals: Beyond electrical conductivities and field-effect transistors (FETs). Angew Chem Int Ed, 2019, 58: 9696-9711

Acknowledgements We are grateful to the National Natural Science Foundation of China (51673151), Natural Science Foundation of Hubei Province (2017CFA002), and the Fundamental Research Funds for the Central Universities (2042017kf0247 and 2042018kf0014) for financial support.

Author contributions Li Q wrote the manuscript with support from Li Z. Both authors contributed general discussion. 


\section{Conflict of interest The authors declare no conflict of interest.}

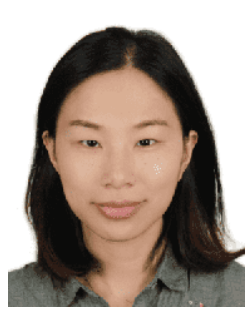

Qianqian Li received her BSc degree from Hubei University, China, in 2004, and then obtained her $\mathrm{PhD}$ degree at Wuhan University in 2009. She is now a full professor at Wuhan University (WHU), and her research interests are in the design and synthesis of new optoelectronic functional materials.

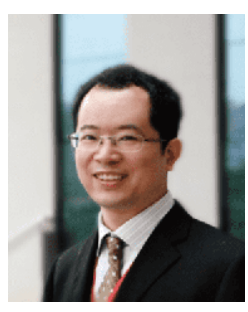

Zhen Li received his $\mathrm{BSc}$ and $\mathrm{PhD}$ degrees from WHU, China, in 1997 and 2002, respectively. In 2003-2004, he worked in the Hongkong University of Science and Technology. In 2010, he worked in Georgia Institute of Technology. He has been a full professor at WHU since 2006 and his research interests are in the development of organic molecules and polymers with new structures and new functions for organic electronics and photonics.

\section{奇妙的分子聚集效应}

李倩倩 ${ }^{1}$, 李振 ${ }^{1,2 *}$

摘要 有机材料的宏观性质是分子聚集效应的客观体现, 不仅取决 于单个分子的结构, 而且与整个分子的聚集形式密切相关. 通过对 分子聚集态行为的有效调控, 科学家们发现了一些完全不同于单 个分子特性的聚集态发光现象, 包括发光强度、颜色、形式以及 激发过程的差异. 本文对这些有趣的聚集态发光材料进行了简要 的综述，系统分析了分子聚集模式和分子间相互作用对材料发光 性能的影响, 并介绍了“MUSIC”的理念, 以音乐创作形象化材料设 计, 强调分子聚集态行为的重要性. 